\title{
Étude du comportement de différents génotypes de rosiers de serre (Rosa hybrida) propagés par bouturage. l. Bouturage et culture de «miniplants» en serre.*
}

\author{
E Berninger 1, R Barrade 1, K Pudelska 2 \\ avec la collaboration technique de G Bauthamy, L Rames, D Sabourin \\ 1 INRA, Station d'amélioration des plantes fiorales, 83600 Fréjus, France; \\ 2 Agricultural Academy in Lublin, Faculty of Horticulture, Institute of Horticultural Production, \\ ul St Leszczy̆nskiego 7, 20-069 Lublin, Pologne
}

(Reçu le 10 novembre 1989, accepté le 19 avril 1990)

\begin{abstract}
Résumé - L'application d'une technique de bouturage rapide de rosiers de serre en conditions contrôlées d'environnement et avec des boutures calibrées, permet d'obtenir à toute époque de l'année des clones de «miniplants» en pots, cultivables ensuite en serres ou en chambres climatisées. Là variation des caractéristiques morphologiques et physiologiques observées sur une collection variétale lors du bouturage et de la première floraison des miniplants en serre, et les relations existant entre caractéristiques des miniplants et d'une culture adulte sont présentées.

Pour obtenir des lots homogènes de boutures puis de miniplants, il faut disposer en même temps d'un nombre suffisant de tiges florifères au même stade de maturité, ce qui limite l'emploi d'une telle technique dans un processus de sélection. Par contre, les observations et notations de caractéristiques importantes sont aisées. La culture de séries successives de miniplants permet, sur des espaces réduits, une approche relativement simple du développement des variétés de roses.
\end{abstract}

rosier de serre / bouturage / milieu contrôlé / génotype / précocité / floraison

Summary - Study of the behaviour of different genotypes of greenhouse rose trees (Rosa hybrida) propagated by cuttage. I. Cutting and growing of miniplants in greenhouses. A simple technique of cuttage of greenhouse roses allows year-round preparation of small "miniplant" clones for pot cultivation in greenhouses or climatic rooms (figs 1,2). Some physiological traits of cuttings and miniplants can vary owing to their position on the mother stem (table I) and to environmental conditions during the cuttage period (table II). In order to reduce the causes of variation, cuttage was carried out in a controlled environment, with standardized size and node level cuttings. Morphological and physiological traits observed in an assortment of genotypes during rooting (table III), and the miniplant crop period in the greenhouse (table $N, V$ ), and the relations between data collected on miniplants and on an adult crop (table VI) are presented. In order to obtain homogeneous batches of cuttings and subsequently of miniplants, a sufficient number of floral stems at the same stage of maturity and available at the same time is required. This prerequisite constitutes a limitation of the technique as a tool in breeding processes. On the other hand, observation and determination of important characteristics do not present problems. With successive crops of miniplants on limited cultivation surfaces it is possible to study in a simple manner the development of rose varieties.

greenhouse rose / cutting / controlled environment / genotype / earliness / flowering

\footnotetext{
"Étude ayant bénéficié d'un soutien financier du conseil régional Provence-Alpes-Côte d'Azur : «Nouveaux génotypes de fleurs”, 1987; et de la CEE : «Recherches physiologiques pour la sélection de plantes plus économes en énergien, 1987-1989.
} 


\section{INTRODUCTION}

Les variétés de rose utilisées pour la production de fleurs coupées en serre présentent de grandes différences de couleur, morphologie, caractères physiologiques. Ces derniers sont importants pour le rendement de la culture et difficilement quantifiables. La culture est pérenne, les mêmes variétés sont cultivées sous des latitudes éloignées et dans des climats très variés. L'aptitude à tolérer un éclairement réduit ou une basse température nocturne est utile en hiver en régions tempérées ou froides. En outre, dans les zones subtropicales d'altitude, où les serres ne sont pas chauffées, les cultures subissent de fortes amplitudes thermiques du jour à la nuit.

En général, l'expérience acquise après plusieurs années de culture sous différentes latitudes permet de classer des variétés pour leur comportement en conditions plus ou moins éclairées ou chauffées, et de connaître leur souplesse d'adaptation, leur productivité, la qualité ou les défauts des fleurs (tenue au stockage, au transport, durée de survie en vase, etc) mais les essais nécessaires sont longs et encombrants. II est difficile de faire cohabiter en serre des types dont les rythmes de production ou les exigences sont différentes. Pour un physiologiste, ces essais sont relativement peu instructifs en raison de la fluctuation des paramètres climatiques qui ne peuvent être répétés dans le détail d'une année à l'autre, et de l'hétérogénéité microclimatique bien réelle dans la plupart des installations de serres.

Le plant de rosier adulte usuellement cultivé est une structure complexe, associant la variété à fleurs et un porte greffe, porteuse de bourgeons et de tiges florifères en compétition nutritionnelle. L'exposition au rayonnement naturel de ces bourgeons et tiges est hétérogène. Les caractéristiques de précocité ou de dimensions des tiges florifères varient en fonction des conditions d'environnement, et elles ne peuvent être établies qu'après de nombreuses observations et mesures.

II est possible de miniaturiser et de simplifier le plant de rosier, en réalisant des "miniplants", à partir de boutures, cultivables en parcelles de dimensions réduites et bien contrôlées en serres ou en chambres climatisées.

La culture en lignes de miniplants de différents génotypes révèle de très nettes différences de développement et de morphologie entre les clones, qu'il est tentant de caractériser par cette technique. Ces caractéristiques sont-elles comparables à celles observées sur des plants adultes, en serre de production ? Tel est l'objet de ce premier article.

L'approche écophysiologique du développement des miniplants demande une maîtrise des principaux paramètres climatiques. Un deuxième article présentera les résultats obtenus en conditions artificielles d'éclairement et de température.

\section{MATÉRIEL ET MÉTHODES}

\section{Matériel végétal}

Les rosiers de serre, Rosa hybrida, tétraploïdes d'origine hybride, sont caractérisés par leurs facultés de floraison remontante, c'est-à-dire continue en conditions d'éclairement non limitant, et en serres moyennement chauffées (à environ $15^{\circ} \mathrm{C}$ la nuit) et par leurs qualités de présentation et de tenue en fleurs coupées. Le matériel utilisé ici est constitué, soit de variétés créées par différents obtenteurs, soit de génotypes issus du programme d'études de notre laboratoire.

\section{Techniques de bouturage et de culture}

La source de boutures est constituée par des tiges florifères droites et, si possible, verticales, produites en serre, au stade normal de récolte commerciale (début d'épanouissement), prélevées en évitant d'abîmer leur feuillage puis immédiatement alimentées en eau (fig 1).

Le bouturage selon la technique développée par Dubois et De Vries (1985) est fait en chambre climatisée éclairée. La bouture est constituée d'un tronçon
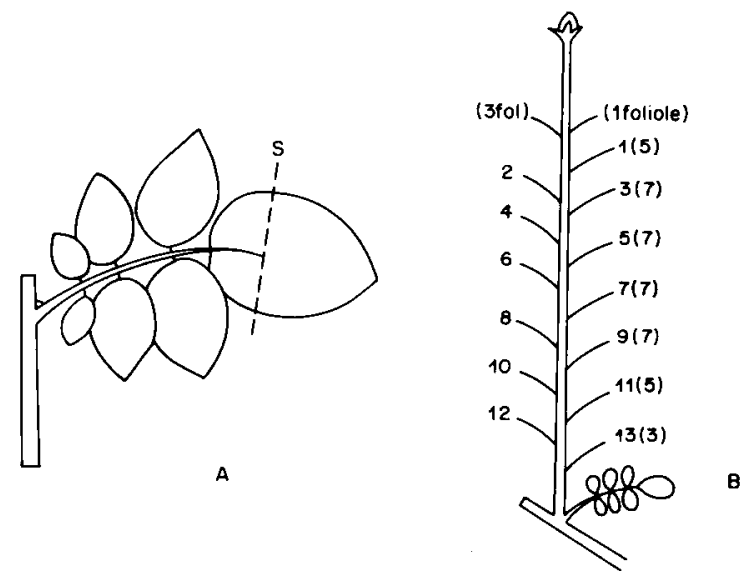

Fig 1. A Bouture feuillée de rosier; $s$ = section éventuelle pour réduction de la surface foliaire). B Tige au stade début d'epanouissement du bouton floral. Numérotation des feuilles et nombre de folioles composant la feuille (entre parenthèses); présentation schématique, la phyllotaxie réelle n'est pas respectée. 
de tige portant un nœud et sa feuille composée (fig 1A).

Pour uniformiser ce matériel de départ, des segments de tige de $5 \mathrm{~cm}$ de long sont coupés, et le feuillage est limité à $60 \mathrm{~cm}^{2}$ environ. Les auteurs précités ont montré que la croissance de la nouvelle pousse et des racines est conditionnée par la surface foliaire de la bouture. En raison des différences de surface des feuilles entières existant entre génotypes, entre tiges, entre feuilles d'une même tige etc, l'intervention est nécessaire. Elle permet aussi d'assurer une disposition régulière et une densité élevée de boutures dans les bacs d'enracinement.

Lors des prélèvements de boutures, le sommet des tiges (étages 1-2-3) est éliminé mais le plus souvent, on ne dispose pas, en même temps, de suffisamment de tiges du même génotype dans le même état pour n'exploiter que 1 ou 2 boutures par tige : les lots sont donc des mélanges de boutures des étages 4 à 10 environ (fig 1B). Les boutures dont le bourgeon a commencé à s'allonger sont éliminées.

Après prélèvement, les boutures sont immergées dans un bain fongicide (Benomyl, 0,75\%), égouttées, et leur base enrobée avec une poudre contenant $0,5 \%$ d'acide indole butyrique AIB. En l'absence d'AIB, la formation des racines est plus lente, leur nombre est réduit.

Elles sont placées à raison de $100-150 / \mathrm{m}^{2}$ dans des bacs garnis d'une couche de $15 \mathrm{~cm}$ de substrat humide tourbe-perlite 50:50 à pH 6. Ces bacs sont ensuite couverts d'un film plastique transparent (bouturage à l'étouffé). L'éclairage est fourni par des lampes à vapeur de sodium haute-pression de $400 \mathrm{~W}$, fonctionnant $20 \mathrm{~h}$ sur 24. Au niveau des boutures, les conditions réalisées sont :

- température $22^{\circ} \mathrm{C}$;

- humidité relative très proche de $100 \%$;

- irradiance PAR 10 à 12 W.m-2.

L'enracinement commence après 2 semaines et est presque total pour la plupart des variétés après 3 semaines. Les boutures sont alors extraites des bacs, notées, triées, et plantées dans des pots de 3 ou 11 , contenant le mélange NTS2 (tourbe enrichie du commerce), gravier de porphyre 50:50. Après la plantation en pots, on parle de «miniplants».

La culture est ensuite faite sur tablettes en serre, à la densité de $35-50$ plants $/ \mathrm{m}^{2}$. Chaque parcelle variétale de 7-10 plants est, si possible, répétée. Des soins d'ombrage et de bassinage pendant les premiers j, peuvent être nécessaires pour ménager une transition entre l'ambiance du bac de bouturage et celle de la serre, beaucoup plus contrastée en éclairement, hygrométrie et température. Par la suite, il suffit d'alimenter les pots en eau par subirrigation, et de tuteurer les pousses si nécessaire.

Le bouturage en blocs de laine de roche de $250 \mathrm{~cm}^{3}$ environ a aussi été réalisé, mais cette technique exige une surveillance de l'alimentation hydrique et minérale plus compliquée.

Afin d'observer l'apparition des racines, des essais ont été faits en maintenant la base des boutures au contact d'un feutre humide (technique «hydroponique»).

\section{Facteurs influençant l'évolution du bourgeon axillaire}

Au moment du bouturage, la majorité des bourgeons axillaires est quiescente, en raison, notamment, des inhibitions hormonales et trophiques exercées par les organes dominants. Une bonne synthèse bibliographique de ce problème a été faite par Van den Berg, 1987. Effectivement, l'état des bourgeons varie selon le stade de maturité des tiges donneuses (l'inhibition est moindre après flétrissement de la fleur), selon les saisons (inhibition moindre au printemps et en été, ou après une période prolongée de températures basses), selon les génotypes, et surtout selon le niveau des bourgeons sur les tiges : l'aspect et le comportement des bourgeons supérieurs, proches de la fleur, ou de ceux de la base, sont différents. L'ontogénèse des bourgeons d'une même tige explique les différences observées (Zamski et al, 1985) : ceux de la base, formés avant la croissance de la tige et fortement inhibés, sont dits proleptiques, ceux proche du sommet formés pendant la période de croissance de la tige, peu ou pas inhibés, sont dits sylleptiques.

\section{Mesures et observations}

\section{Données climatiques}

Dans les chambres de bouturage, à régulation thermostatique, un thermohygrographe étalonné permet de contrôler les températures d'air de la photophase et de la scotophase, et de calculer la moyenne journalière.

Dans les serres, il y a acquisition continue des températures d'air (sondes Pt 100 en abris ventilés). L'irradiance est estimée à partir des données de pyranomètres extérieurs et de coefficients saisonniers de transmission du rayonnement.

\section{Notations sur boutures et miniplants}

Au cours du bouturage, on note essentiellement la mortalité, l'allongement du bourgeon axillaire, l'enracinement à l'arrachage.

En cours de culture, pour chaque miniplant, on note les dates des stades suivants :

- premiers symptômes d'allongement de bourgeon (dp);

- pousse mesurant $1 \mathrm{~cm}(1 \mathrm{~cm})$;

- bouton floral ou sépales visibles (B);

- fleur récoltable (F).

Les autres caractéristiques observées ont été :

- avortement de la pousse = croissance ralentie puis arrêtée avant apparition du bouton floral (un taux 
élevé d'avortement résulte de conditions insuffisantes d'éclairement ou de température en début d'allongement).

- nombre de feuilles composées formées avant le bouton (= «nombre de nœuds»);

- longueur, poids frais et sec de la tige au stade de récolte;

- présence et nombre de départs de la base ("gourmands») à la floraison.

\section{Essals réalisés}

\section{Variété Sonia}

La variété Sonia Meilland ( $R$ ), cv Sweet Promise, référence habituelle des études physiologiques et agronomiques à travers le monde, a été utilisée pour préciser la technique de bouturage et de culture, puis pour vérifier l'uniformité des bacs de bouturage, des serres expérimentales et enfin, comme témoin dans tous les essais de génotypes.

\section{Types représentatifs de la variabilité des rosiers}

Une douzaine de types supposés représentatifs de la variabilité des rosiers de serre ont été essayés tous les 3 mois pendant 2 ans consécutifs. Les résultats présentés ont été obtenus dans des essais à 2 répétitions de 7 miniplants par génotype en pots de $3 \mathrm{I}$, soit initialement 24 boutures, en photopériode de $20 \mathrm{~h}$ sur 24.

\section{Génotypes de l'essai basse température}

Soixante génotypes faisant partie d'un essai de comportement à «basse" température $\left(11^{\circ} \mathrm{C}\right.$ nocturne) en serre de production et culture en plein sol, ont également été cultivés en miniplants, et les corrélations entre observations faites dans ces 2 cultures ont été calculées.

\section{RÉSULTATS ET DISCUSSION}

\section{Comportement au bouturage}

Un essai de comportement en boutures et en miniplants de Sonia illustre les potentialités des bourgeons de différents étages (tableau I). Ceux des étages supérieurs (1-3) débourrent le plus facilement puis différencient le bouton floral après émission d'un nombre réduit de feuilles, alors que ceux de la base (10-12) débourrent tardivement et irrégulièrement.

L'évolution précoce d'un bourgeon axillaire dépend aussi des conditions de bouturage (tableau II, l'effectif minimal est de 72 boutures par essai) :

Un débourrement précoce est favorisé, - par un éclairement réduit ou une scotopériode longue; ainsi, le pourcentage de boutures débourrées au bout de $20 \mathrm{j}$ après éclairement continu est de $11 \%$ (moyenne de 2 essais); il atteint en moyenne $65 \%$ après éclairement de $20 \mathrm{~h}$ sur 24 ou de 16 h sur 24;

- par la disponibilité en eau du substrat : les observations montrent que le pourcentage de pousses est toujours plus important en bouturage sur laine de roche $(82,84,100 \%)$ que sur substrat tourbe-perlite (50-56\%).

Le débourrement est ralenti par l'apport d'AIB (il atteint 66 et $85 \%$ dans des lots sans hormone, contre $50 \%$ dans un lot témoin avec poudrage AIB 0,5\%).

La "population" de boutures dans la plupart de nos essais est un mélange de boutures des étages 4-7 ou supérieurs à 7, selon les génotypes. Cette population paraît homogène pour le processus d'enracinement, (observé en «hydroponique» et vérifié par des prélèvements

Tableau 1. Comportement de boutures prélevées à différents niveaux. cv Sonia bouturé le 13 janvier, transplanté en

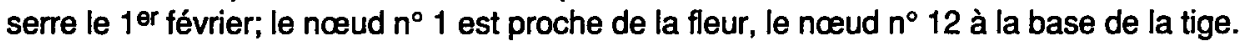

$\begin{gathered}\text { Position des } \\ \text { boutures de boutures } \\ \text { (nœud) }\end{gathered}$
$\begin{gathered}\text { Nbre de boutures } \\ \text { poussées le } \\ \text { O1 février }\end{gathered}$

Evolution ultérieure en miniplants

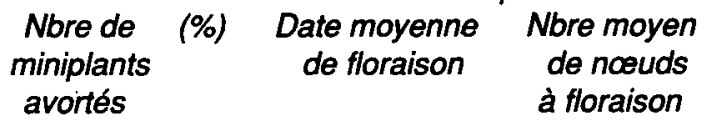

\begin{tabular}{|c|c|c|c|c|c|c|c|}
\hline 1 à 3 & 20 & 10 & (50) & 6 & (30) & 17 mars & 6 \\
\hline 4 à 9 & 49 & 17 & (35) & 15 & (30) & 21 mars & 7.5 \\
\hline 10 à 12 & 14 & 2 & (14) & 1 & (7) & 25 mars & 8 \\
\hline
\end{tabular}


Tableau II. Comportement de boutures de Sonia placées en différentes conditions de photopériode.

\begin{tabular}{|c|c|c|c|c|c|c|c|c|}
\hline $\begin{array}{c}\text { Date } \\
\text { de bouturage }\end{array}$ & 02 septembre & 27 novembre & 25 février & 25 mai & 27 août & 05 décembre & 03 mars & 02 juin \\
\hline Photopériode & $24 \mid$ & $7 / 24$ & $16 \mathrm{~h}$ & & & $20 \mathrm{~h}$ & & \\
\hline \% débourrés & 14 & 8 & 95 & 36 & 33 & 50 & 80 & 68 \\
\hline$\%$ enracinés & 98 & 100 & 100 & 100 & 90 & 99 & 100 & 100 \\
\hline
\end{tabular}

dans les bacs de substrat) mais non pour celui de débourrement (fig 2). L'examen des courbes montre que la période d'enracinement coïncide avec l'arrêt du débourrement, lequel reprend après transplantation hors du bac de bouturage : il doit y avoir compétition entre les racines et les pousses pour les réserves limitées disponibles. L'effet inhibiteur de l'acide indole butyrique sur les pousses pourrait s'expliquer comme une conséquence temporaire de son rôle rhizogène.

Les caractéristiques de débourrement du bourgeon axillaire et de développement radiculaire au $18^{\ominus}-20^{\circ}$ j de bouturage, établies pour 11 génotypes après 9 essais aux différentes époques de l'année, sont résumées dans le tableau III (l'effectif minimal est de 24 boutures par lot et par essai).

Les pourcentages de débourrement sont aussi variables que ceux de Sonia présentés au tableau II, et l'établissement de valeurs moyennes n'a guère de sens. La distinction de types à débourrement facile ou rapide, moyen, lent, et à enracinement abondant ou rapide, moyen, faible ou lent, par rapport au témoin Sonia lui-même à débourrement et enracinement rapides est suffi-

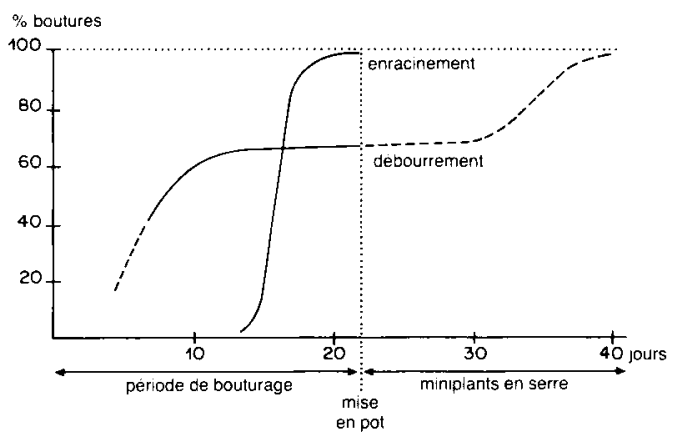

Fig 2. Évolution du débourrement du bourgeon axillaire et de l'enracinement d'un lot «moyen» de boutures (étages 4-9), cv Sonia; présentation schématique d'un essai avec relevé journalier du débourrement en bac puis en serre.

sante, et ces comportements génotypiques ont été confirmés dans les essais ultérieurs.

\section{Evolution de l'apex}

En serre, la différenciation florale de l'apex débute après différenciation de quelques ébauches foliaires, lorsque la pousse mesure 1-2 cm (Moe

Tableau III. Comportement de 11 génotypes au bouturage. Enracinement et débourrement jugés du $18^{e}$ au $20^{e} \mathrm{j}$. Moyenne de 9 essais.

\begin{tabular}{llll}
\hline Génotype & Enracinement & Débourrement & \% débourrés en fin de bouturage \\
\hline Sonia & rapide & rapide & 48 \\
Indira & rapide & rapide & 46 \\
Frefair & rapide & rapide & 34 \\
$2-121-1$ & moyen & rapide & 52 \\
$0-166-2$ & très lent & rapide & 54 \\
Lancome & rapide & moyen & 23 \\
$2-98-5$ & rapide & moyen & 23 \\
$0-188-5$ & lent & moyen & 29 \\
$2-34-7$ & rapide à moyen & lent & 5 \\
$2-162-5$ & lent & lent & 12 \\
Madelon & lent & lent & 11 \\
\hline
\end{tabular}


et Kristoffersen, 1969; Horridge et Cockshull, 1974; Cockshull et Horridge, 1977). En cas d'éclairement insuffisant, au sens trophique, le bourgeon avorte. Or, dans les conditions d'éclairement imposées lors du bouturage $\left(10 \mathrm{~W} . \mathrm{m}^{-2}\right.$, $20 \mathrm{~h} / \mathrm{j}$ ), le rayonnement journalier atteint $720 \mathrm{~kJ}$, bien inférieur à l'éclairement hivernal moyen en serre. Cette insuffisance est probablement aggravée par une température moyenne élevée et une surface foliaire réduite.

Ainsi, chez Sonia, la majorité des pousses ayant atteint ou dépassé $1 \mathrm{~cm}$ avant la transplantation en conditions favorables, avorteront. Les lots de boutures de certains génotypes sont entièrement débourrés avant la fin du bouturage, ce qui compromet leur utilisation en l'état, mais la suppression de la nouvelle pousse apicale entraîne l'activation de bourgeons latéraux improprement appelés "stipulaires" (fig 3).

L'opération consiste à casser la jeune pousse tendre, ou à la couper à ras si les tissus sont déjà durcis. Les 2 premiers bourgeons "stipulaires» débourrent en une semaine environ, et il faut en supprimer 1 dès que possible. Les potentialités des pousses axillaires et stipulaires, dans nos essais, sont peu différentes : en principe, le bourgeon axillaire est à l'origine plus gros et plus différencié que les stipulaires.

Pour Sonia, dans les mêmes conditions, favorables dès le début de sa croissance, il évolue un peu plus vite (1 journée sur 30-50) et différencie parfois une feuille composée de moins avant floraison.

La synchronisation du débourrement des bourgeons axillaires restés quiescents au cours du bouturage est moins bonne que celle des stipulaires, probablement stimulée de façon brutale par la suppression au même moment des pousses axillaires. Pour la pratique des essais,

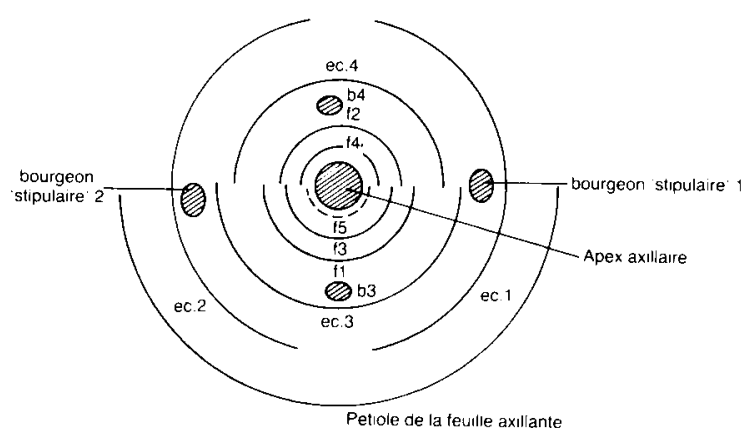

Flg 3. Diagramme en coupe transversale d'un bourgeon axillaire de rose cv Sonia, $7^{\theta}-9^{\theta}$ nceud; $e c=$ écaille, $f=$ feuille composée, b = bourgeon. avec des pousses axillaires, il faut disposer d'effectifs supplémentaires destinés à remplacer quelques miniplants $(10-15 \%)$ dont le débourrement reste bloqué.

L'exploitation des bourgeons stipulaires paraît susceptible d'être généralisée, mais elle entraîne des délais de culture un peu plus longs, et oblige à exciser une partie des pousses.

\section{Comportement des miniplants en serre}

Les résultats présentés dans les tableaux IV et $V$ sont établis, sauf précision supplémentaire, à partir de miniplants issus de pousses axillaires.

\section{Sonia}

Le comportement saisonnier de Sonia et les principales données climatiques sont résumés au tableau IV.

En conditions climatiques "moyennes" de printemps, avec une température proche de $20{ }^{\circ} \mathrm{C}$, les phases successives du développement durent approximativement :

- 0 - 8j de plantation à dp;

$-5-10$ j de dp à $1 \mathrm{~cm}$;

$-14 \mathrm{j}$ de $1 \mathrm{~cm}$ à $\mathrm{B}$;

-21 j de $B$ à $F$.

Les variations saisonnières de la précocité de floraison, exprimées par la durée de la période $d p-F$, sont en relation évidente avec les conditions thermiques et, secondairement, avec les conditions d'éclairement. Ces résultats sont en bon accord avec ceux présentés par Van den Berg (1987), pour la même variété.

\section{Génotypes choisis pour représenter la variabilité des rosiers}

Le comportement de 11 génotypes bouturés à 4 dates différentes est résumé au tableau $\mathrm{V}$.

Dans l'ensemble, chaque variété est bien caractérisée comme précoce (Sonia, Frefair, 2-121-1, 2-162-5, 2-98-5) ou tardive (Madelon, 234-7, 0-166-2), avortant peu (la majorité) ou beaucoup (2-121-1), a croissance forte (Indira, Lancôme, 2-98-5) ou réduite (0-166-2, Madelon, 2-121-1, 2-34-7). Pour la précocité de floraison, qui semble être la donnée la plus fiable, des interactions génotypes $x$ saison, ou changements de classement des génotypes, apparaissent (exemples : Madelon en hiver, 2-98-5 en été). 
Tableau IV. Variation saisonnière de 3 caractéristiques des miniplants, cv Sonia, et de 2 paramètres climatiques de la culture en serre.

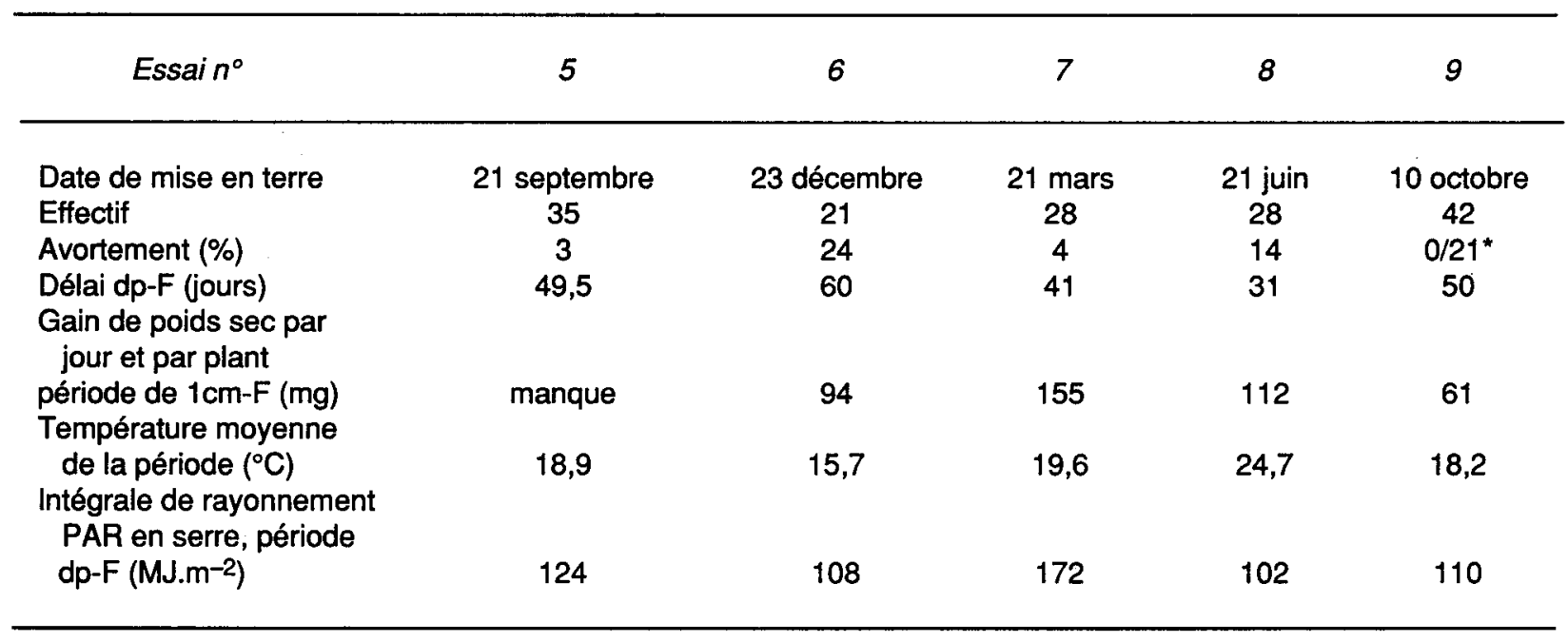

" miniplants «stipulaires», avortement 0 sur 28 ; miniplants axillaires, avortement 3 sur 14.

L'avortement élevé de quelques lots au printemps semble lié à des difficultés de reprise des miniplants introduits dans une serre non blanchie en période de très beau temps.

Le gain journalier de poids $\mathrm{sec}$, déterminé par des prélèvements destructifs lors de la maturité individuelle, est indicatif de la vigueur de croissance. Le classement des génotypes varie selon les essais. Le nombre de nœuds varie peu entre essais, et la donnée moyenne est seule présentée. Le rapprochement des caractéristiques de bouturage et de culture ne montre pas de rela-

Tableau V. Principales caractéristiques de miniplants de 11 génotypes, en serre chauffée à environ $13^{\circ} \mathrm{C}$ la nuit.

\begin{tabular}{|c|c|c|c|c|c|c|c|c|c|c|c|c|}
\hline \multirow[b]{2}{*}{$\begin{array}{l}\text { Essai } \\
\text { Variétés ou } \\
\text { génotypes }\end{array}$} & \multicolumn{4}{|c|}{$\begin{array}{l}\text { Précocité (dp-F) } \\
\text { Nombre de jours }\end{array}$} & \multicolumn{4}{|c|}{$\begin{array}{l}\text { Avortement } \\
\text { (\%) }\end{array}$} & \multicolumn{3}{|c|}{$\begin{array}{c}\text { Poids sec/ } \\
\text { Nbre de jours (mg/j) }\end{array}$} & \multirow{2}{*}{$\begin{array}{c}\text { Nombre } \\
\text { de nouuds } \\
\text { Moyenne } \\
\text { des } 4 \text { essais }\end{array}$} \\
\hline & i 5 & 6 & 7 & 8 & 5 & 6 & 7 & 8 & 6 & 7 & 8 & \\
\hline Sonia & 49,5 & 60 & 41 & 31 & 3 & 24 & 4 & 14 & 94 & 155 & 112 & 8,8 \\
\hline Lancome & 53 & 65 & 51 & 32,5 & 0 & 0 & 7 & 0 & 85 & 160 & 157 & 9,3 \\
\hline Indira & 54 & 63,5 & $49^{*}$ & 37 & 0 & 0 & $21^{*}$ & 0 & 103 & $174^{*}$ & 182 & 11,2 \\
\hline Frefair & 48,5 & 63 & 47,5 & 31,5 & 0 & 7 & 21 & 7 & 82 & 152 & 141 & 8,4 \\
\hline $2-34-7$ & 57 & 65,5 & 52 & 35 & 14 & 14 & 7 & 0 & 84 & 112 & 142 & 10,7 \\
\hline $2-98-5$ & 50 & 62 & $41^{\star}$ & 34 & 0 & 0 & $0^{*}$ & 0 & 84 & $186^{*}$ & 159 & 8,4 \\
\hline $2-121-1$ & 1 & 57 & 41,5 & $30,5^{\star}$ & 86 & 75 & 43 & $7^{*}$ & 71 & 169 & $132^{*}$ & 6,8 \\
\hline $2-162-5$ & 49,5 & 60 & 41 & 32 & 0 & 0 & 7 & 0 & 79 & 144 & 194 & 8,4 \\
\hline Madelon & 59 & 63,5 & 55,5 & 37,5 & 0 & 0 & 0 & 7 & 83 & 118 & 145 & 10,3 \\
\hline $0-188-5$ & 54,5 & 62 & $46^{*}$ & 32,5 & 7 & 7 & $14^{*}$ & 0 & 85 & $146^{*}$ & 168 & 9,5 \\
\hline $0-166-2$ & 62 & $74^{*}$ & I & $37^{\star}$ & 0 & $0^{\star}$ & 1 & $7^{\star}$ & $68^{*}$ & 1 & $118^{\star}$ & 9,8 \\
\hline Moyenne & 54 & 63 & 47 & 34 & & & & & 83 & 151 & 150 & \\
\hline
\end{tabular}

Essai 5 = date de plantation automne 21 septembre; Essai 6 = date de plantation hiver 23 décembre; Essai 7 = date de plantation printemps 21 mars; Essai 8 = date de plantation été 21 juin;

"lots «stipulaire». 
tion entre la vitesse de débourrement au bouturage et la précocité de floraison. Un système radiculaire très lent ou réduit lors de la transplantation correspond à, ou entraîne, un retard de croissance de la pousse qui se trouve bientôt ombrée par celles des plantes voisines (cas de 0-166-2).

La seule relation notable entre caractéristiques mesurées sur miniplants relie la précocité de floraison et le nombre de nœuds, faible chez les types précoces, élevé chez les types tardifs ( $n=+0,732$, significatif à $P=0,05$ mais non à $P=0,01$ (seuil 0,735 ).

\section{Comparaison de miniplants et de rosiers adultes cultivés en plein sol}

Soixante génotypes multipliés par bouturage, puis plantés en janvier 1988, en plein sol dans une serre de sélection, sont formés pendant les 8 mois suivants puis soumis, après une coupe réglée en fin novembre 1988, à un chauffage réduit à $11^{\circ} \mathrm{C}$. Dans ces conditions, la remontée hivernale de fleurs est limitée et lente : 70-90 j jusqu'à récolte. On a noté la précocité de floraison (de la coupe à la date moyenne de récolte), la longueur des tiges et le nombre de nœuds, la production de fleurs de premier choix par parcelles de 7 plants.
Pour les miniplants, en raison des limitations de place, 31 de ces génotypes ont été bouturés fin novembre 1988, à partir des tiges récoltées en serre de sélection avant la remontée d'hiver. Les miniplants et leur pousse axillaire ont été observés en culture à $13^{\circ} \mathrm{C}$ au minimum, de midécembre à mi-février. Ils évoluent plus vite que la remontée des plants adultes cultivés à une température nocturne de $11^{\circ} \mathrm{C}$ (essai 34 , tableau VI).

Vingt-huit autres génotypes ont été bouturés en mars 1989 , à partir de tiges de l'essai adulte $11^{\circ} \mathrm{C}$. Leur culture en miniplants, conduite sur bourgeons stipulaires en raison d'un débourrement généralisé au bouturage, a eu lieu en avrilmai (essai 39, tableau VI).

Les caractéristiques des miniplants de ces 2 essais ont été comparées à celles de la remontée hivernale des plantes adultes, par les coefficients de corrélation $r$ (tableau VI).

Le mauvais comportement en miniplants des génotypes peu enracinés en fin de bouturage, déjà noté, se confirme dans l'essai 34 , mais n'apparaît pas dans l'essai 39 où l'enracinement est, en général, bon. On a noté que 4 des 5 génotypes peu enracinés de la série 34 sont aussi parmi les moins productifs de l'essai adulte, pour la remontée étudiée.

Dans les comparaisons entre miniplants et culture adulte, on note de grandes différences de

Tableau VI. Valeur et signification des cœefficients $r$ de corrélation entre caractéristiques des tiges fleuries de miniplants et d'une remontée de plantes adultes.

$\begin{array}{cc}\text { Miniplants d'hiver } & \text { Miniplants d'avril-mai } \\ 31 \text { génotypes } & 28 \text { génotypes } \\ \text { (éssai 34) } & \text { (essai 39) }\end{array}$

$r$ Précocité en miniplants

$r$ Longueur des tiges miniplants

$r$ Nombre de nceuds miniplants

$r$ Production des miniplants (nombre de tiges fleuries)

$r$ Production des miniplants (nombre de tiges fleuries)

$r$ vigueur des miniplants (accroissement journalier de matière sèche)

seuils $r$ à $P=0,05$

seuils $r$ à $P=0,01$
- Précocité en remontée d'hiver

- Longueur en remontée d'hiver

- Nombre de nœuds en remontée d'hiver

- Production en remontée d'hiver (nombre de tiges) (1)

- Production en remontée d'hiver (nombre de tiges $x$ longueur) (2)

- Production en remontée d'hiver (expression 1 ou 2)

$\begin{array}{cc}+0,75 & \text { nul } \\ +0,59 & +0,57 \\ +0,71 & +0,71 \\ \text { nul } & \text { nul } \\ \text { nul } & \text { nul } \\ \text { nul } & \text { nul }\end{array}$

0,35

0,37

0,45 
longueur et de poids des tiges au stade récolte. Pour Sonia, on a couramment des longueurs de $60 \mathrm{~cm}$ (adulte) et $30 \mathrm{~cm}$ (miniplants) et des poids frais de $30-35 \mathrm{~g}$ (adulte) et $15-20 \mathrm{~g}$ (miniplants), alors que le nombre de nœuds et la précocité de floraison, à conditions climatiques égales, sont peu différents.

La précocité de floraison, la longueur des tiges et le nombre de nœuds concordent bien ou assez bien, d'une condition à l'autre, mais aucun des caractères observés sur miniplants ne peut être formellement relié à la production observée des plantes adultes en serre : pour ces derniers, le nombre potentiel de pousses dépend de la ramification très variable des individus et des génotypes, et les caractéristiques de tolérance à la température réduite ont sans doute beaucoup joué dans la production réalisée. Nous ne disposons pas, au stade miniplants, d'éléments indicatifs de ces caractéristiques. Le pourcentage de réussite (non avortement) noté sur 14 miniplants ne semble pas en relation avec la production hivernale des plantes adultes en conditions de chauffage réduit.

\section{DISCUSSION GÉNÉRALE}

Au cours du processus classique de création variétale, des notations de précocité de floraison et de qualité des tiges florales sont possibles:

- sur plants de semis, mais ceux-ci sont souvent trop serrés ou d'espacement irrégulier, en compétition, non synchrones;

- en pépinière de plein air après greffage, mais les tiges et fleurs formées en plein air en été sont très compactes et soumises à des agressions variées; cette possibilité est encore peu explorée;

- sur plants clonés en serre; les délais de multiplication et d'implantation en serre retardent ces notations d'environ un an, par rapport à la première floraison des plants de semis.

Le bouturage en conditions contrôlées et la culture standardisée de miniplants offrent des possibilités intéressantes, mais il faut en attendre des précisions sur un nombre limité de génotypes déjà triés pour d'autres critères, plutôt qu'un crible précoce de nombreux semis. Toutefois, un bon comportement sur leurs propres racines peut être recherché très tôt pour des variétés destinées aux cultures hors-sol.
A la différence de pousses formées sur un plant adulte porteur de réserves abondantes, les pousses de miniplants disposent de peu de réserves et d'un appareil foliaire initial réduit. Les différences génotypiques de vigueur des jeunes pousses, exprimées en gains de poids journalier par exemple, sont très nettes. Elles peuvent dépendre de la capacité photosynthétique de la feuille axillante, et des capacités de puits du bourgeon et de la jeune pousse. La technique décrite ici devrait permettre d'aborder de telles études.

\section{CONCLUSION}

Plusieurs caractères sont aisément observables et quantifiables sur boutures et miniplants. Certains sont des critères d'aptitudes physiologiques (mise à fleur, précocité, etc.) ou de qualité de la production (longueur des tiges), et leurs relations avec les facteurs du climat sont nettes. Ces relations ne doivent pas masquer l'importance des effets propres de la bouture (incluant sa position sur la tige, sa surface foliaire, probablement son diamètre, la saison de prélèvement, les conditions culturales antérieures), ni celles des effets du protocole de bouturage lui-même, sur le comportement ultérieur des miniplants. En premier lieu, il convient de maîtriser le débourrement en bac de bouturage si l'on veut faire des essais sur pousse axillaire.

Le choix des conditions de bouturage n'est pas arbitraire, mais guidé par le souci d'une réussite rapide et par des impératifs de simplicité technique, comme l'absence de fertilisation du substrat. Ces particularités peuvent handicaper un génotype en essai comparatif, alors qu'elles seraient corrigées dans une opération limitée à ce seul génotype (cas de réactions à l'hormone $A \mid B$, de noircissement pathologique du bois bouturé, d'enracinement lent, etc.) mais il serait vain de vouloir optimiser les conditions appliquées à un ensemble de types ayant une bonne diversité physiologique.

Dans l'état actuel, ces essais de miniplants paraissent pouvoir compléter ou conforter les opérations classiques de jugement et de tris sur plants adultes pratiquées dans des conditions délibéremment optimales ou limitantes en serre. 


\section{RÉFÉRENCES}

Cockshull KE, Horridge JS (1977) Apical dominance and flower initiation in the rose. $J$ Hortic Sci 42, 412-427

Dubois LAB, de Vries DO (1985) Onderzoek met stekken var "Sonia" (Expériences sur les boutures de "Sonia"). Vakblad voor de Bloemisterij 45, 26-31

Horridge JS, Cockshull KE (1974) Flower initiation and development in the glasshouse rose. Sci Hortic $2,273-284$
Moe R, Kristoffersen T (1969) The effect of temperature and light on growth and flowering of Rosa "Baccara" in greenhouses. Acta Hortic 14, 157-167

Van den Berg GA (1987) Influence of temperature on bud break, shoot growth, flower bud atrophy and winter production of glasshouse roses. Thèse, Pudoc, Wageningen, $170 \mathrm{p}$

Zamski E, Oshri S, Zieslin N (1985) Comparative morphology and anatomy of axillary buds along a rose shoot. Bot Gaz 146-2, 208-212 